\title{
Supporting Conference Attendees with Visual Decision Making Interfaces
}

$\begin{array}{ll}\begin{array}{l}\text { Katrien Verbert } \\ \text { Department of Computer }\end{array} & \begin{array}{l}\text { Denis Parra } \\ \text { Dcience }\end{array} \\ \begin{array}{l}\text { KU Leuven } \\ \text { Science } \\ \text { Leuven, Belgium } \\ \text { katrien.verbert@cs.kuleuven.be }\end{array} & \begin{array}{l}\text { School of Engineering, PUC } \\ \text { Chile } \\ \text { dparra@ing.puc.cl }\end{array} \\ \begin{array}{l}\text { Peter Brusilovsky } \\ \text { School of Information Sciences }\end{array} & \text { Bruno Cardoso } \\ \text { University of Pittsburgh } & \begin{array}{l}\text { Science } \\ \text { Pittsburgh, PA, USA }\end{array} \\ \text { peterb@pitt.edu } & \text { KU Leuven } \\ & \text { Leuven, Belgium } \\ & \text { bruno.cardoso@cs.kuleuven.be }\end{array}$

Chirayu Wongchokprasitti

School of Information Sciences

University of Pittsburgh

Pittsburgh, PA, USA

chw20@pitt.edu

Permission to make digital or hard copies of part or all of this work for personal or classroom use is granted without fee provided that copies are not made or distributed for profit or commercial advantage and that copies bear this notice and the full citation on the first page. Copyrights for third-party components of this work must be honored. For all other uses, contact the owner/author(s).

Copyright held by the owner/author(s).

IUl'17 Companion, March 13-16, 2017, Limassol, Cyprus

ACM 978-1-4503-4893-5/17/03.

http://dx.doi.org/10.1145/3030024.3038273

\begin{abstract}
Recent efforts in recommender systems research focus increasingly on human factors affecting recommendation acceptance, such as transparency and user control. In this paper, we present IntersectionExplorer, a scalable visualization to interleave the output of several recommender engines with user-contributed relevance information, such as bookmarks and tags. Two user studies at conferences indicate that this approach is well suited for technical audiences in smaller venues, and allowed the identification of applicability limitations for less technical audiences attending larger events.
\end{abstract}

\section{Author Keywords}

Interactive visualization; recommender systems; set visualization

\section{ACM Classification Keywords}

H.5.2 [Information interfaces and presentation (e.g., $\mathrm{HCl}$ )]:

User Interfaces

\section{Introduction}

In recent years, the research community has become increasingly aware of the fact that the effectiveness of recommender systems goes beyond traditional metrics like recommendation accuracy [5]. Thus, research on human factors is gaining momentum and several user-centric ap- 


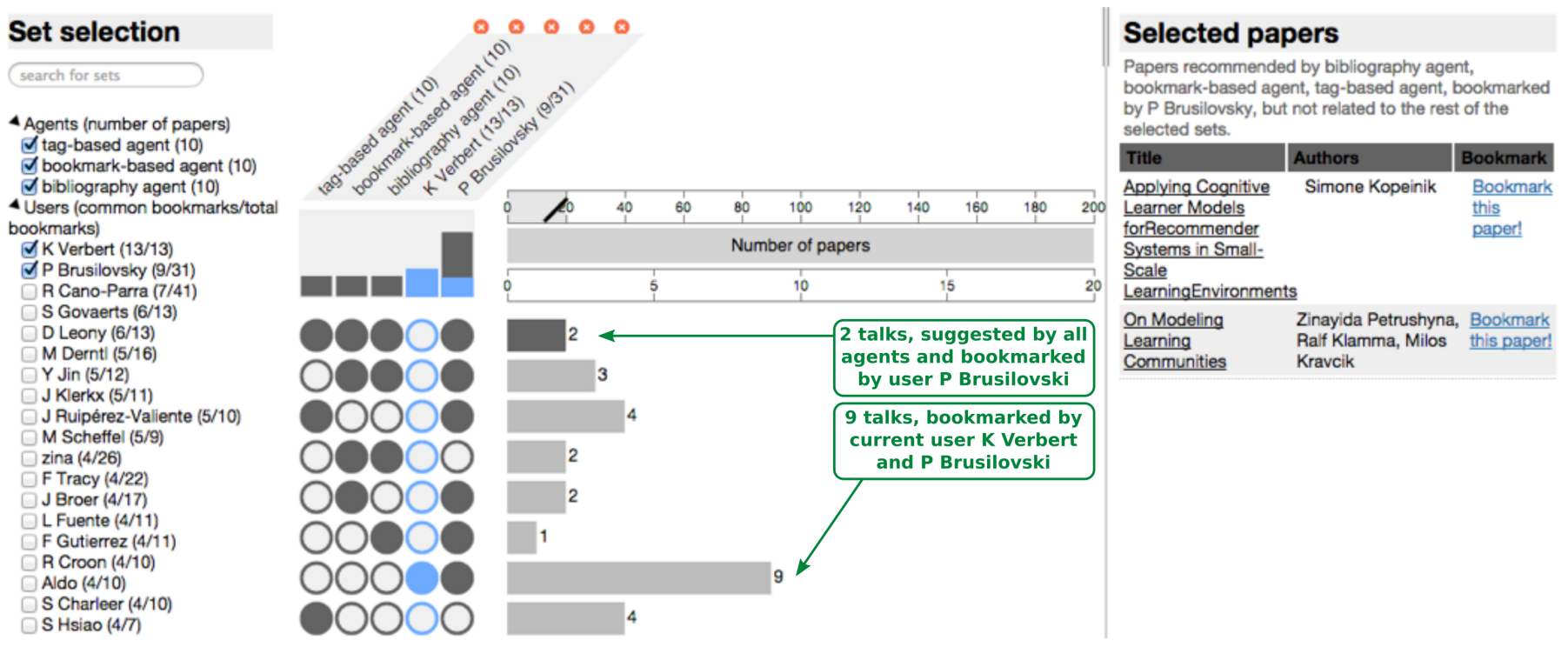

Figure 1: IntersectionExplorer visualizes relationships between recommendations generated by multiple recommendation techniques (agents) and bookmarks of users and tags to increase relevance of recommendations. Note: explanatory callouts in green are not part of IEx's interface.

proaches have been explored over the past decade. In particular, a combination of interactive visualization methods with recommendation techniques to improve the acceptance of recommendations emerged as a visible trend that was explored in our recent survey [3]. Interactive visual recommendation approaches address the "black box" issue by allowing users to have an insight into the system's inner logic, thereby leveraging the transparency of the recommendation process. Prominent examples are TasteWeights [1] and SetFusion [6]. Both systems elicit preference data from users at run-time in order to adapt recommendations, and allow users to fine-tune the weights of a hybrid recommender system that merges the outcome of different recommendation techniques.
The system we present in this paper, IntersectionExplorer(IEx), further extends this concept by offering a visualization of the relationships between different relevance-bearing entities, including user-contributed data such as user bookmarks and tags. The novelty of IEx is twofold: first, it uses a set-based relevance approach that is not limited to merging the output of multiple recommendation techniques. In contrast to the majority of earlier work on visual recommendation, IEx combines several types of relevance-bearing entities (users, tags, and recommendation agents) to increase richness and relevance of recommendations. Second, it uses a simplified version of UpSet [4], a recently proposed set-based visualization approach, to present relationships between different entities in a scalable way. 


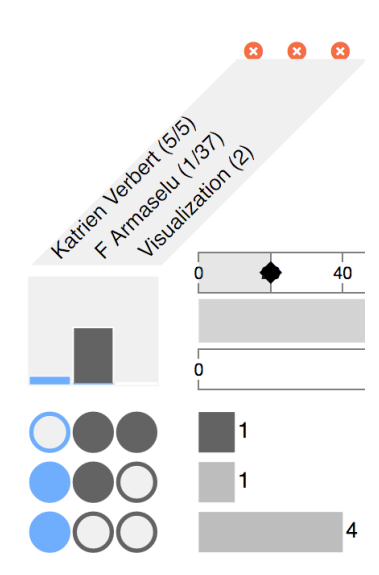

Figure 2: In this image, the first row filters out those talks that are bookmarked by user "F Armaselu" and also tagged with "Visualization".
The results of our user studies suggest that this approach, when used by a technical audience, effectively encourages users to explore related items and ultimately increases the perceived relevance of the recommendations. At the same time, we discovered that the approach has limitations when used by a non-technical audience in diverse settings.

\section{IntersectionExplorer (IEx)}

The goal of IEx - to help users find the items that are most interesting for them while also allowing them to understand and control the recommendation process - is not trivial. To address the visual complexity that inevitably comes from the amount of information involved, IEx uses a simplified version of UpSet [4], a matrix-based visualization technique to represent sets and overlaps between these sets. The scalable set-based nature of IEx enables it to present information from several recommender engines as well as to combine and visually represent the relationships between several types of relevance-bearing entities (users, tags, and recommendation agents) in a matrix view. An overview of IEx's user interface is shown in Figure 1. It is separated in three parts. In the left part, the user can select different entities (agents, users and tags) and interactively explore associated sets of suggested items in the intersection area (interface central part). Depending on the entities selected, the added sets will be composed of the talks recommended by agents, those bookmarked by other users or talks tagged by a specific tag. In the central part of the interface, columns represent sets of suggestions from different entities (the current user is highlighted in blue) and rows represent commonalities between them, signalled by filled cells. Finally, the right part of the interface displays the details of the selected set items.

One of the biggest advantages of UpSet matrix-based set visualization is scalability. Whereas a Venn diagram can only display the intersections of a limited number of sets, the UpSet technique can present many sets in parallel, as only a single column has to be added to add another set to the visualization. This greatly reduces space requirements while increasing the information density.

The visualization enables users to explore the overlaps of user bookmarks, recommendations of agents and tags as a basis to find relevant items. The first row of Figure 1 for instance represents two talks that are recommended by three agents and that are also bookmarked by "P Brusilovsky".

The second row represents three talks that are recommended by two different agents and also bookmarked by the same user. The key idea is that these overlaps can be used as additional relevance indicators: talks that are selected by multiple agents and also bookmarked by a known user can potentially have higher relevance. Similar overlaps can be explored with tags. Figure 2 presents an example where the talks of the active user ("K Verbert") and a known user to her ("F Armaselu") are presented in parallel to talks marked with the tag "Visualization". The first row enables the user to filter out those talks bookmarked by " $F$ Armaselu" and also tagged with "Visualization" as a basis to find relevant talks. Similar relationships can be explored to talks recommended by specific agents to filter out the potentially more relevant recommendations.

\section{Evaluation}

In order to evaluate IEx, we have deployed it on top of Conference Navigator 3 (CN3) [2]. CN3 is a social, personalized web-based system that supports academic conference attendees and suggests talks using different recommendation engines. IEx metaphorizes these engines as entities (the agents) and places their top-10 suggestions at the same level as user bookmarks and tags, thus allowing to explore how these automatic recommendations relate to those 
based on user input. Interface complexity often emerges as one of the critical factors limiting their applicability. Therefore, to better assess IEx's usability and applicability, we conducted two user studies in different contexts. The first was performed at EC-TEL conference, an event focused on technology enhanced learning with a relatively small, highly technical audience (20 participants; mean age: 32.9 years; SD: 6.32; female: 3). In turn, the second study was conducted at the Digital Humanities 2016 Conference, a large event with a broader, less technology-oriented audience ( 37 participants; mean age: 38 years; SD: 10; female: 11). In both studies, users were asked to explore the IEx visualization freely and to bookmark five relevant items.

The results of the first user study show a positive effect of the IEx approach on recommendation effectiveness, when used in a relatively small event, with a highly technical audience. More specifically, recommendation effectiveness was significantly higher when users explored the combination of an agent with a user or tag than when they explored agents only [7]. This trend was not observed in the results of the second study, performed at a larger event with a less technically oriented audience. This study revealed points that need to be addressed for the approach to be an effective tool in such settings. The most important challenge is that in large and diverse settings, the matrix easily becomes very sparse. Increasing the typical top-10 limit of recommendation agents may be a solution, as it will increase the chance of overlap between the agent suggestions and bookmarks or tags. The identified issues will be addressed in our future work, which will include also additional studies to explore the applicability of the IEx in diverse settings.

\section{Acknowledgments}

Part of this work was supported by the KU Leuven Research Council (grant agreement no. STG/14/019).

\section{REFERENCES}

1. Svetlin Bostandjiev, John O'Donovan, and Tobias Höllerer. 2012. TasteWeights: a visual interactive hybrid recommender system. In Proc. RecSys'12. ACM, 35-42.

2. Peter Brusilovsky, Jung Sun Oh, Claudia López, Denis Parra, and Wei Jeng. 2016. Linking information and people in a social system for academic conferences. New Review of Hypermedia and Mult. (2016), 1-31.

3. Chen He, Denis Parra, and Katrien Verbert. 2016. Interactive recommender systems: A survey of the state of the art and future research challenges and opportunities. Expert Systems with Applications 56 (2016), 9-27.

4. Alexander Lex, Nils Gehlenborg, Hendrik Strobelt, Romain Vuillemot, and Hanspeter Pfister. 2014. UpSet: visualization of intersecting sets. Visualization and Computer Graphics, IEEE Transactions on 20, 12 (2014), 1983-1992.

5. Sean M McNee, John Riedl, and Joseph A Konstan. 2006. Being accurate is not enough: how accuracy metrics have hurt recommender systems. In $\mathrm{CHI} O 6$ EA. ACM, 1097-1101.

6. Denis Parra and Peter Brusilovsky. 2015.

User-controllable personalization: A case study with SetFusion. International Journal of Human-Computer Studies 78 (2015), 43-67.

7. Katrien Verbert, Karsten Seipp, Chen He, Denis Parra, Chirayu Wongchokprasitti, and Peter Brusilovsky. 2016. Scalable exploration of relevance prospects to support decision making. In Proc. of the Joint Workshop on Interfaces and Human Decision Making for Recommender Systems co-located with ACM RecSys 2016. CEUR-WS, 28-35. 